\title{
Cosmic ray mass composition with LOFAR
}

\author{
S. Buitink ${ }^{* 1}$, A. Bonardi ${ }^{2}$, A. Corstanje ${ }^{2}$, H. Falcke ${ }^{2,3,4}$, B. M. Hare ${ }^{5}$, J. R. Hörandel ${ }^{1,3}$, \\ P. Mitra ${ }^{1}$, K. Mulrey ${ }^{1}$, A. Nelles ${ }^{2,6}$, J. P. Rachen ${ }^{2}$, L. Rossetto ${ }^{2}$, P. Schellart ${ }^{2,8}$, \\ O. Scholten ${ }^{5,7}$, S. ter Veen ${ }^{2,4}$, S. Thoudam ${ }^{2,9}$, T. N. G. Trinh ${ }^{5}$, and T. Winchen ${ }^{1}$
}

${ }^{1}$ Astrophysical Institute, Vrije Universiteit Brussel, Pleinlaan 2, 1050 Brussels, Belgium

${ }^{2}$ Department of Astrophysics / IMAPP, Radboud University, P.O. Box 9010, 6500 GL Nijmegen, The Netherlands

${ }^{3}$ NIKHEF, Science Park Amsterdam, 1098 XG Amsterdam, The Netherlands

${ }^{4}$ Netherlands Institute of Radio Astronomy (ASTRON), Postbus 2, 7990 AA Dwingeloo, The

Netherlands

${ }^{5}$ KVI-CART, University Groningen, P.O. Box 72, 9700 AB Groningen, The Netherlands

${ }^{6}$ Department of Physics and Astronomy, University of California Irvine, Irvine, CA 92697-4575, USA

${ }^{7}$ Interuniversity Institute for High-Energy, Vrije Universiteit Brussel, Pleinlaan 2, 1050 Brussels, Belgium

${ }^{8}$ Department of Astrophysical Sciences, Princeton University, Princeton, NJ 08544, USA

${ }^{9}$ Department of Physics and Electrical Engineering, Linné Universitetet, 35195 Växjö, Sweden

E-mail: Stijn.Buitinkevub.be

The LOFAR radio telescope measures the radio emission from extensive air showers with unprecedented precision. In the dense core individual air showers are detected by hundreds of dipole antennas. By fitting the complex radiation pattern to Monte Carlo radio simulation codes we obtain measurements of the atmospheric depth of the shower maximum $X_{\max }$ with a precision of $<20 \mathrm{~g} / \mathrm{cm}^{2}$. This quantity is sensitive to the mass composition of cosmic rays.

We discuss the first mass composition results of LOFAR and the improvements that are currently being made to enhance the accuracy of future analysis. Firstly, a more realistic treatment of the atmosphere will decrease the systematic uncertainties due to the atmosphere. Secondly, a series of upgrades to the LOFAR system will lead to increased effective area, duty cycle, and the possibility to extend the composition analysis down to the energy of $10^{16.5} \mathrm{eV}$.

35th International Cosmic Ray Conference - ICRC2017

10-20 July, 2017

Bexco, Busan, Korea

${ }^{*}$ Speaker. 


\section{Introduction}

Radio detection of air showers [1] is a rapidly developing technique, that offers promising possibilities to accurately measure the air shower energy and the atmospheric depth of the shower maximum, known as $X_{\max }[2,3]$. The technique can be used in combination with established techniques such as fluorescence detection and surface detection. Dense antenna arrays like the core of LOFAR [4], or the low-frequency part of the future SKA observatory [5], are useful for detailed verification of our understanding of the emission mechanism and the accuracy of radio simulation codes. Moreover, they allow $X_{\max }$ measurements with a precision of $<20 \mathrm{~g} / \mathrm{cm}^{2}$ or even better [6]. The dense antenna distribution comes at the expense of a limited instrumented area, which leaves a study of ultra-high-energy cosmic rays up to the cut-off out of reach. However, air shower measurements with LOFAR and SKA are of particular interest for mass composition studies at energies between the second knee and the ankle [7, 8].

LOFAR is a digital radio telescope constructed in the Netherlands with satellite stations across Europe. It consists of thousands of dipole antennas, operating in the frequency range of $10-$ $240 \mathrm{MHz}$. It produces extremely detailed air shower radio data, by using the dense core region, or superterp, where 384 antennas are located within a circle of 320 m diameter. A particle array, LORA [9], has been installed on the superterp and is used for triggering and reconstruction. Each antenna contains a ring buffer which is read-out in case of a trigger. The full waveform is stored for offline analysis [10].

The radio emission of air showers results from a combinations of geomagnetic and charge excess radiation. To correctly calculate it, it is also needed to take into account the relativistic propagation effects due to the small but finite refractivity of air (see references in [1]). The interference of these contributions causes an azimuthal asymmetry of the distribution of radio power on the ground. It is also the cause of the location-dependent polarization angle [11] and even a small but measurable amount of circular polarization [12]. To reach a high precision reconstruction of the air shower a two-dimensional fit is developed that takes into account the asymmetrical nature of the emission [3]. The first LOFAR composition results based on this method are shown in Fig. 2 [13].

The largest systematic uncertainty in the LOFAR $X_{\max }$ reconstruction is due to effects of the variation of the index of refraction in air $[13,14,16]$. In previous studies, we relied on CORSIKA [17] simulations using the US Standard Atmosphere. Furthermore, the radio emission code CoREAS [18] internally uses a linear scaling of the refractivity with air density, only accepting a variable ground level value for the index of refraction as input. In reality though, the refractivity depends on the relative humidity, which can vary significantly with altitude.

In order to reduce the uncertainties, we have developed a tool that extracts realistic profiles for the air density and index of refraction as a function of atmospheric depth. An upcoming release of CORSIKA will include this code as well as adaptations that make it easy to run CORSIKA/CoREAS with these additional input files. We have revised our simulation pipeline and plan to soon start new simulation production based on this and other improvement. In these proceedings we describe the updated simulation pipeline and other future improvements to LOFAR cosmic-ray detection. 


\section{Simulation pipeline}

Reconstruction of air showers with LOFAR is based on the production of dedicated sets of simulations for each detected high-quality air shower ${ }^{1}$. There are two reasons for this. Firstly, the shape of the radio profile on the ground depends not only on the azimuth and zenith angle, but also on the angle with the geomagnetic field. Parametrizations of these profiles exists but will not yield the same resolution on $X_{\max }$ that full simulations provide [19].

Secondly, LOFAR antennas are not placed on a regular grid but in dense rings with spacings in between. This makes it nearly impossible to construct criteria on the energy, core location and arrival direction for which a $100 \%$ detection efficiency is reached. Instead of defining such criteria, we evaluate for each measured shower whether or not the detection efficiency is near $100 \%$. This is done by creating a set of CORSIKA showers that have the same energy, arrival direction and core position, but cover the natural range of $X_{\max }$ values. Only if all showers in the set trigger LORA and pass the high-quality criterium, the shower is included in the mass composition analysis.

The number of simulations needed to reconstruct the shower and evaluate a possible composition bias is optimized with CONEX [20], which allows us to quickly produce the longitudinal profiles for hundreds of showers. Now, it is possible to select a subset of these showers for subsequent full CORSIKA simulation. We choose this subset by first selecting the two proton showers with the highest and lowest value for $X_{\max }$. Then, we select nine more proton showers with intermediate values, (nearly) equally spaced between the extreme values. Since the range of $X_{\max }$ values for iron showers is much narrower, we select a total of five iron showers using the same algorithm. This procedure assures that the simulation set can be used for the bias test.

Finally we add eleven additional shower simulations close to the estimated $X_{\max }$ of the measured shower to increase the resolution of the reconstruction. This estimation is given by fitting a parametrization of the radio signal [19] to the data, that has a resolution of $\sim 40 \mathrm{~g} / \mathrm{cm}^{2}$.

Another advantage of having dedicated sets of simulations per shower is that we can use atmospheric description that closely match the conditions during the actual measurement, instead of profiles that are averaged over years or months. In particular, the radio emission is sensitive to fluctuations in the humidity which change over much shorter time scales. The up-to-date profiles are based on data from GDAS [15] and a detailed description of the procedure can be found elsewhere in these proceedings [16].

The radio emission is simulated for antennas in a star-shaped pattern in the shower plane. The LOFAR low-band antenna model is applied to the simulated electric fields to calculate the measured signals in the two dipoles. The measured pulse power is defined as the total power in a $55 \mathrm{~ns}$ window centered around the pulse maximum, summed over both polarisations. Finally, a two-dimensional map of the pulse power is created by interpolating the star-shaped pattern. More details are found in [3].

A GEANT4 simulation is used to calculate the lateral distribution of the energy deposited in the LORA scintillators. These simulations are needed to test whether all simulated showers would indeed trigger the LORA detector. They are also included in the shower reconstruction.

\footnotetext{
${ }^{1}$ High-quality currently means that at least four rings of LOFAR low-band antennas should be able to independently reconstruct the shower direction that is in agreement with the LORA particle reconstruction. However, this requirement could be relaxed in the future.
} 


\section{Reconstruction}
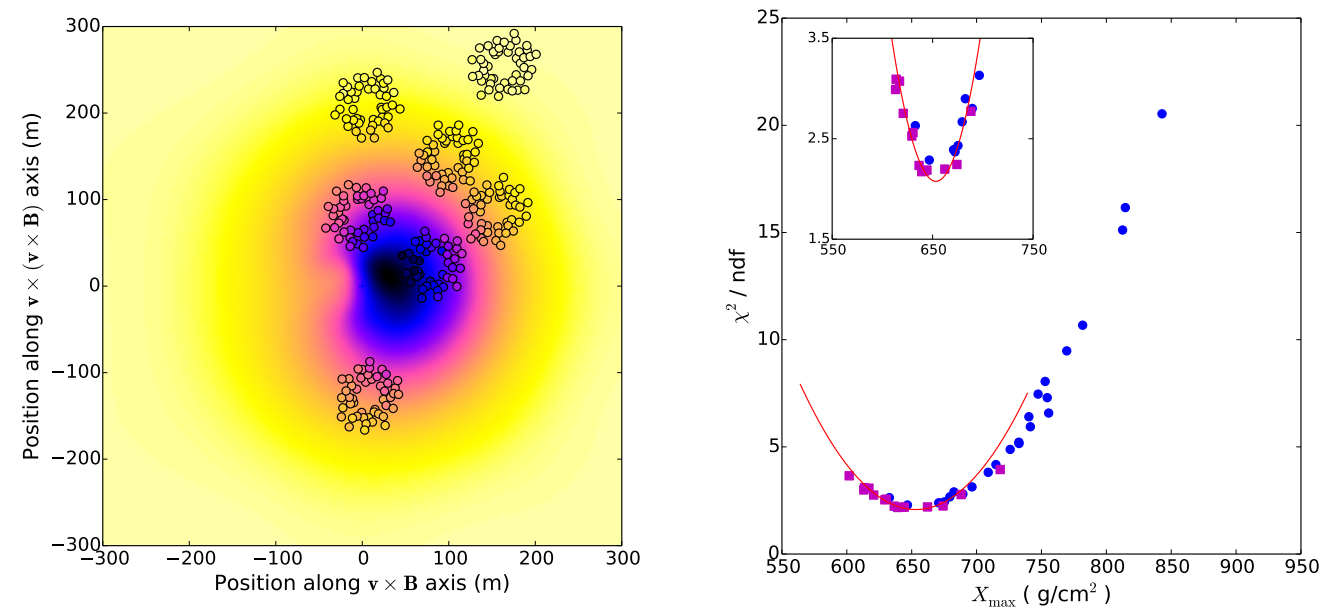

Figure 1: Left: event reconstruction. The small circles indicate LOFAR antenna positions (grouped in larger circles) projected into the shower plane. Their color indicates the measured power of the radio pulse. The background is the two-dimensional simulated radio power map. The fit quality is high when the colors inside the circles blend into the background. Right: reconstruction of $X_{\max }$. Every data point corresponds to a single simulated shower. Together they form the dedicated simulation set produced for this particular shower. The reduced $\chi^{2}$ of the fit is plotted against $X_{\max }$ and a parabola is fitted to find the optimum $X_{\max }$. The inlay is a zoomed-in version of the minimum of the curve demonstrating the its sharpness. Purple squares correspond to iron showers and blue circles to proton showers.

The radio and particle profiles are fitted to the data simultaneously. The fit has four free parameters: two for the shower core position and scaling parameters for both the radio and the particle profiles. Inclusion of the particle data in the fit does not have much effect on the fitted core position in practice. However, it acts as an additional consistency check and sets the energy scale. The analysis presented here does not yet include the absolute calibration of the antennas [21] so the scaling parameter for the radio power has an arbitrary value. In effect, the ratio of two scaling parameters also has an arbitrary but constant value. The width of the distribution of this parameter is a measure for the energy resolution of the reconstruction, which is $32 \%$.

The reduced $\chi^{2}$ of the fit is very sensitive to $X_{\max }$ as is seen in Fig. 1. The minimum of the $\chi^{2}$-curve is found by fitting a parabola to the points near the lowest value. The curve is not smooth because shower-to-shower fluctuations other than variations in $X_{\max }$. In order to obtain a robust parabola fit, data points that have neighbours on both sides that have a lower $\chi^{2}$-value are excluded from the fit. Monte Carlo simulations have shown that this produces a significant increase in $X_{\max }$ resolution.

\section{Results}

The two main results from the first LOFAR composition analysis are shown in Fig. 2. The left panel displays the LOFAR $X_{\max }$ measurements in comparison with other experiments based 

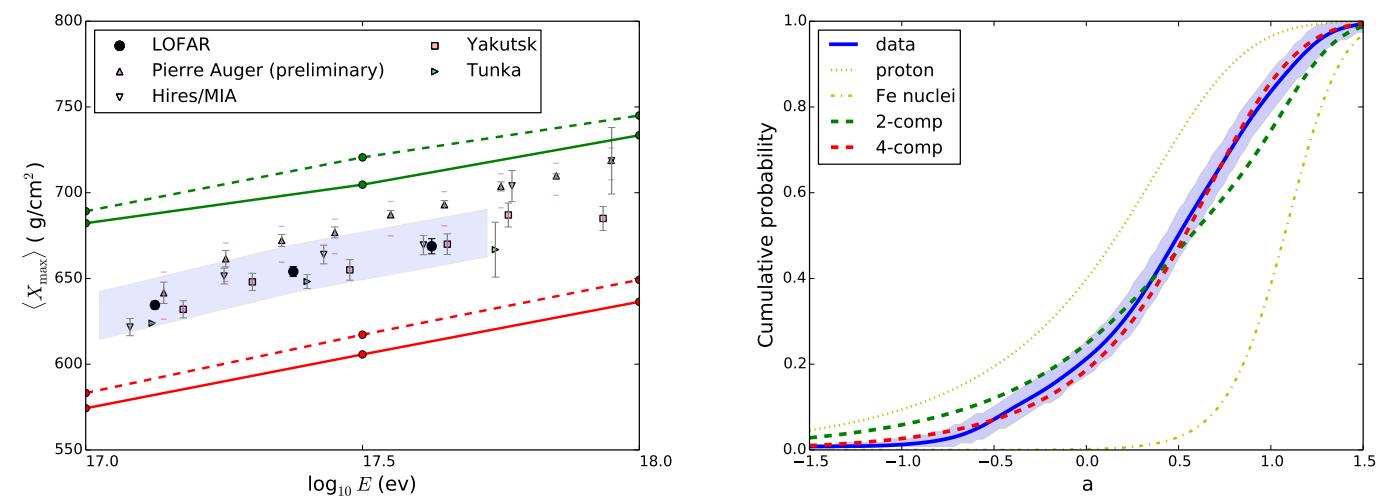

Figure 2: Left: mean $X_{\max }$ as a function of energy. The LOFAR results are in agreement with results based on different techniques within systematic uncertainties (Auger systematic uncertainties are indicated with purple bars [22]). The green lines are for pure proton composition and the red lines for pure iron (dashed: EPOS-LHC, solid: QGSJETII). Right: cumulative distribution of $a$ for LOFAR data (blue; with $1 \sigma$ uncertainty band) and different compositions. A four-component model is required to reproduce the data.

on other techniques. The error bars represent $1 \sigma$ statistical uncertainties and the shaded region indicates the systematic uncertainty.

The right panel shows the cumulative distribution of $a$, a parameter that indicates the position of the $X_{\max }$ value of a particular shower relative to the simulated mean for pure proton $(a=0)$ and pure iron $(a=1)$ lines. A pure proton composition would yield a broad distribution of $a$ around $a=0$, while a pure iron composition corresponds to a narrow distribution around $a=1$. The measured distribution can now be fitted with multi-component models. The minimum number of components required to achieve a good fit is four (proton, helium, nitrogen, iron). A full scan of the allowed parameter space yields a large contribution of light elements. Although there is a large uncertainty in the contribution of individual elements, the total fraction of light elements $(\mathrm{p}+\mathrm{He})$ is well constrained. It has a best fit value of 0.8 and lies within the range $[0.38,0.98]$ at a $99 \%$ confidence level for QGSJETII. More details are found in [13].

\section{Systematic uncertainties due to the atmosphere}

As mentioned above, the systematic uncertainty on $X_{\max }$ is currently dominated by atmospheric uncertainties. Fluctuations in air density and humidity reduce the resolution on $X_{\max }$. Moreover, since the original simulations used an index of refraction on ground level that corresponds to $0 \%$ relative humidity, the reconstructed value for $X_{\max }$ is expected to shift to larger values for realistic atmospheres. In the original analysis, this led to an asymmetric total systematic uncertainty of $+14 /-10 \mathrm{~g} / \mathrm{cm}^{2}$.

Interestingly, another contribution to the total systematic uncertainty is an observed zenith dependence on the mean value of $X_{\max }$. Since this can also be the result of using inaccurate atmospheric profiles, this dependence may reduce for the new simulations based on GDAS profiles. Indeed, this would be a confirmation that the new profiles are more realistic than the older. A more detailed account on the various systematic uncertainties is found in [13]. 


\section{Future extensions}
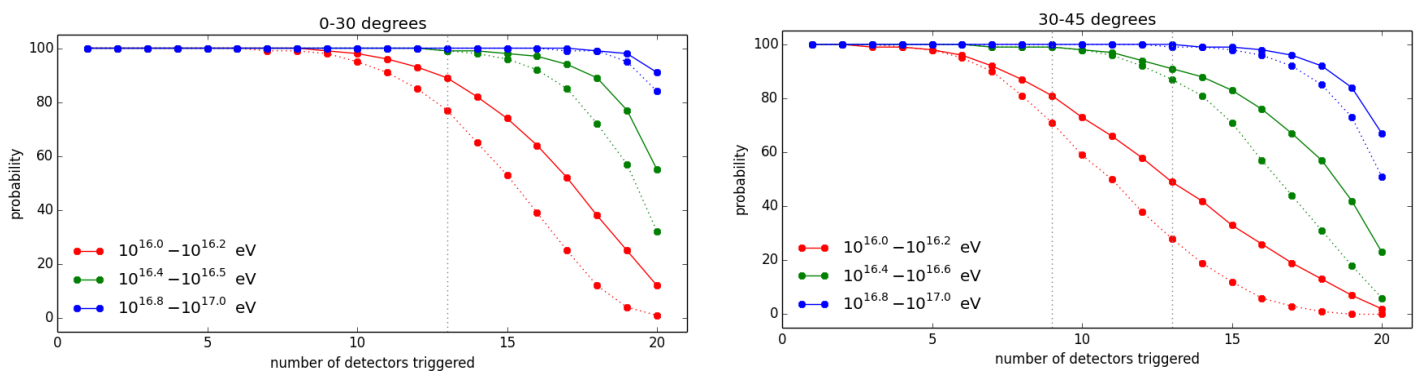

Figure 3: Detection probability for proton showers (solid) and iron showers (dotted) for different energies and zenith angles, as a function of number of particle detectors required for a trigger. In a hybrid triggering scheme, a detection efficiency of $100 \%$ down to $10^{16.5} \mathrm{eV}$ is achieved by reducing the amount of required triggers. The precise amount depends on the area under consideration. For this plot only showers having a core inside the superterp, i.e. within $160 \mathrm{~m}$ of the LORA core, are considered.

The improved simulation pipeline is expected to yield more accurate measurements of $X_{\max }$, but the composition analysis still suffers from low statistics. For the (near) future, several upgrades are planned that will increase the number of unbiased showers.

- LORA expansion Twenty more particle detectors will be installed at five LOFAR stations (rings of antennas) near the superterp. This will both increase the effective area by a factor of $\sim 40 \%$ and allow triggering on a specific class of showers for which the superterp is located outside of the Cherenkov angle. Here, the radio signal quickly becomes weaker but may be more sensitive to the precise longitudinal evolution of a shower. The data will be used to study the possibility of determining more shower evolution parameters than only $X_{\max }$, which could both be useful for composition studies and constraining hadronic interaction models. This can be regarded as a first step towards shower radio tomography, which has been proposed for $\mathrm{SKA}^{2}$. More details on the expansion are found elsewhere in these proceedings [23]

- Hybrid triggering The current triggering system requires 13 out of 20 LORA detectors to trigger. This has two disadvantages. First, while this setting yields a near $100 \%$ detection efficiency for showers above $10^{17} \mathrm{eV}$, most of the triggers are from showers that are of much lower energy and do not produce an observable radio pulse. Indeed, in $80 \%$ of the data that is stored, no radio signal is found. Secondly, nearly half of the high-quality showers for which we can reconstruct $X_{\max }$ fall in the $10^{16.5}-10^{17} \mathrm{eV}$ energy range, where the detection efficiency is too small to allow for an unbiased composition study.

Both problems are resolved simultaneously by implementing a hybrid triggering system that combines information from the particles detectors and radio antennas in real-time. Through continuous monitoring of each antenna data stream it is possible to send trigger messages to the central LORA computer for each radio peak that is above the noise background by a

\footnotetext{
${ }^{2}$ Apart from being a larger and more regularly spaced array, SKA-low has the additional advantage that it will be sensitive to smaller sub-structures in the shower because of its increased frequency range (50-350 MHz).
} 
certain threshold value. In the LORA computer the messages of all antennas are collected and combined with the data from the particle detectors. A hybrid trigger is issued when both a minimum number of antennas and a minimum number of particle detectors show a signal-over-threshold.

Since every hybrid trigger has a visible radio signal we boost our fraction of useful data from $20 \%$ to nearly $100 \%$. At the same time, the risk of triggering on manmade impulsive signals is extremely low since we require coincidence with a particle signal. Even more importantly, we can now lower the required number of particle detectors for a trigger. This means we will be able to detect cosmic ray below $10^{17} \mathrm{eV}$ bias-free, reducing our energy threshold for the mass composition analysis.

Figure 3 shows a simulation study of the detection probability for proton showers (solid lines) and iron showers (dotted lines) for different energy ranges. A bias-free sample is achieved when the proton and iron lines overlap, which only happens when both species reach a probability of nearly $100 \%$. This preliminary study indicates the energy range for mass composition studies can be extended to at least $10^{16.5} \mathrm{eV}$, by reducing the required number of detector for triggering.

- LOFAR 2.0 An often advertised advantage of radio detection of air showers is the larger duty cycle of nearly $100 \%$. However, due to technical and organizational aspects, LOFAR reaches a duty cycle of only 10-20\%. Although air shower detection always run in the background of astronomical observations, there are gaps between these observations. Moreover, more than half of the observations use the high-band antennas, which are of very limited use to air shower analysis [24]. LOFAR 2.0 is a planned upgrade to the observatory that will allow a more simultaneous measurement modes. From a technical side, it will become possible to use low and high band antennas at the same time. Furthermore, observation scheduling will done in a manner that takes advantage of the possibilities of a digital radio telescope. Observations will be able to run in parallel with different start and end times. These changes will make it possible to perform low-band air-shower measurements continuously, improving the duty cycle considerably.

\section{Conclusions}

LOFAR measures the atmospheric depth of the shower maximum $X_{\max }$ with a precision of $<20 \mathrm{~g} / \mathrm{cm}^{2}$ using a technique based on two-dimensional reconstruction of the radiation profile. The first mass composition results are in agreement with measurements based on other techniques and consistent with a strong light-mass component below the ankle. To further optimize the precision and reduce systematic uncertainties, a new simulation pipeline is being developed that incorporates realistic atmospheric profiles. Furthermore, future upgrades are planned that improve the performance of cosmic ray detection with LOFAR, increasing the effective area and duty cycle, and reducing the energy threshold for mass composition analysis. 


\section{Acknowlegdements}

The LOFAR cosmic ray key science project acknowledges funding from an Advanced Grant of the European Research Council (FP/2007-2013) / ERC Grant Agreement n. 227610. The project has also received funding from the European Research Council (ERC) under the European Union?s Horizon 2020 research and innovation programme (grant agreement No 640130). We furthermore acknowledge financial support from FOM, (FOM-project 12PR3041-3) and NWO (Top Grant 614001-454, and Spinoza Prize SPI 78-409). AN is supported by the DFG (research fellowship NE 2031/1-1). LOFAR, the Low Frequency Array designed and constructed by ASTRON, has facilities in several countries, that are owned by various parties (each with their own funding sources), and that are collectively operated by the International LOFAR Telescope foundation under a joint scientific policy.

\section{References}

[1] T. Huege, Physics Reports 620, 1-52 (2016).

[2] W. Apel et al. [LOPES], Phys. Rev. D 90, 062001 (2014).

[3] S. Buitink et al. [LOFAR], Phys. Rev. D 90, 082003 (2014).

[4] M. P. van Haarlem et al. [LOFAR], Astron. \& Astrophys. 556, A2 (2013).

[5] T. Huege et al. [SKA High Energy Cosmic Particle Focus Group], AASKA14, 148 (2015).

[6] A. Zilles, S. Buitink, and T. Huege, these proceedings (2017).

[7] K.-H. Kampert and M. Unger, Astropart. Phys. 35, 660 (2012).

[8] S. Thoudam et al., Astron. \& Astrophys. 595, A33 (2016).

[9] S. Thoudam et al., [LOFAR], Nucl. Instr. and Meth. in Phys. Res. A 767, 339 (2014).

[10] P. Schellart et al. [LOFAR], Astron. \& Astrophys. 560, A98 (2013).

[11] P. Schellart et al. [LOFAR], J. Cosmology Astropart. Phys. 10, 014 (2014).

[12] O. Scholten et al. [LOFAR], Phys. Rev. D 94, 103010 (2016).

[13] S. Buitink et al. [LOFAR], Nature 531, 70-73 (2016).

[14] A. Corstanje et al. [LOFAR], Astropart. Phys. 89, 23 (2017).

[15] NOAA Air Resources Laboratory (ARL), Global Data Assimilation System (GDAS1) Archive Information, Tech. rep. (2004).

[16] P. Mitra et al. [LOFAR], these proceedings (2017).

[17] D. Heck, Report FZKA 6019 (1998).

[18] T. Huege, M. Ludwig, and C. James, AIP Conference Proceedings 1535, 128-132 (2012).

[19] A. Nelles et al. [LOFAR], J. Cosm. Astropart. Phys. 5, 18 (2015).

[20] T. Bergmann et al., Astropart. Phys. 26, 420-432 (2007).

[21] A. Nelles et al. [LOFAR], JINST 10, P11005 (2015).

[22] A. Porcelli et al. [Pierre Auger Collaboration], PoS (ICRC2015) 420; arXiv:1509.03732 (2015).

[23] K. Mulrey et al. [LOFAR], these proceedings (2017).

[24] A. Nelles et al. [LOFAR], Astropart. Phys. 65, 11 (2015). 